\title{
Bases génétiques de la polarité planaire
}

> Certains épithéliums présentent une polarité François Schweisguth orientée perpendiculairement à l'axe de polarité apico-basale des cellules épithéliales. Les bases génétiques et moléculaires de cette polarité planaire ont été analysées depuis une vingtaine d'années chez la drosophile. Des travaux récents montrent que la mise en place de la polarité planaire repose sur la distribution unipolaire d'un petit nombre de molécules de signalisation au cortex apical des cellules épithéliales. La distribution unipolaire de ces molécules définit deux domaines corticaux, opposés et complémentaires, dont l'orientation stéréotypée confère une polarité à l'échelle du tissu. La coordination globale de l'orientation de ces domaines corticaux est assurée par une seconde classe de molécules de signalisation qui forment un gradient d'activité à l'échelle du tissu. La conservation de la mouche aux vertébrés - des molécules de polarité planaire suggère une implication de ces molécules dans la biologie des épithéliums chez les mammifères. <

La plupart des cellules épithéliales présentent une polarité apico-basale évidente. Elles présentent parfois également une polarité dont l'axe est schématiquement orienté perpendiculairement à l'axe apico-basal. Cette seconde polarité, appelée polarité planaire, décrit l'orientation vectorielle d'une cellule épithéliale, ou d'une structure multicellulaire simple, dans le plan d'un épithélium. Ainsi, les poils épidermiques qui couvrent la cuticule des insectes sont tous orientés selon l'axe du corps. Il en est de même des écailles des poissons, des plumes des oiseaux ou des cellules auditives dans l'oreille interne des mammifères. Ces exemples illustrent la grande cohérence de la polarité planaire présentée par chacune des cellules d'un même tissu, d'où le nom de polarité tissulaire, synonyme de polarité planaire. Cette cohérence suggère que les cellules communiquent entre elles pour adopter une même orientation vecto-

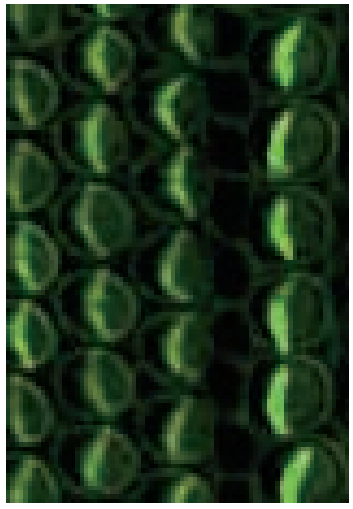

rielle dans le plan de l'épithélium et/ou répondent de manière

École Normale Supérieure, CNRS UMR 8542, 46, rue d'Ulm, 75230 Paris Cedex France. schweisg@wotan.ens.fr convergente à un signal de polarisation agissant sur l'ensemble du tissu. Ces observations soulèvent plusieurs questions intéressantes. Comment est établie une polarité perpendiculairement à l'axe de polarité apicobasale des cellules épithéliales? Quels sont les mécanismes moléculaires qui sous-tendent la polarisation planaire des cellules épithéliales? Comment la direction dans laquelle les cellules s'orientent est-elle déterminée ? Quel est le degré de coordination (ou d'autonomie) de chaque cellule au sein d'un épithélium dans l'adoption d'une orientation planaire? Plusieurs études récentes menées chez la drosophile permettent d'ébaucher des réponses à ces questions $[1,2]$.

\section{Polarisation planaire à l'échelle d'une cellule épithéliale}

La cuticule de la drosophile est recouverte de fines extensions en forme de poil qui sont sécrétées par les cellules épidermiques qui forment un épithélium monocouche à la surface de l'organisme (Figure 1). Dans l'aile, chaque cellule épidermique sécrète une seule extension, appelée poil épidermique. Cette structure, riche en microfilaments, est formée durant la métamorphose au pôle distal de chacune des cellules de l'aile [3]. II en résulte que chaque poil épidermique présente une orientation proximo-distale à la surface de l'aile. La 


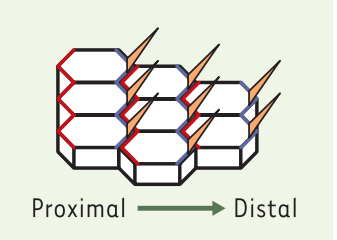

Figure 1. Polarité planaire dans l'aile de drosophile. Les cellules épidermiques hexagonales de l'aile présentent une polarité planaire orientée selon l'axe proximo-distal. Chaque cellule synthétise à son pôle distal un poil orienté vers l'extrémité distale de l'aile. La distribution polarisée des protéines Strabismus et Prickle (pôle proximal, rouge) et celle des protéines Frizzled et Dishevelled (pôle distal, en bleu) définissent deux domaines corticaux opposés.

polarité planaire de cet épithélium est donc aisément visualisable. Cette propriété a facilité l'identification de mutations affectant la polarité planaire dans ce tissu (Tableaul) $[1,4]$. Les mutations inactivant la fonction des gènes frizzled, dishevelled, prickle, strabismus, flamingo ou diego conduisent à la formation du poil épidermique en position centrale à l'apex de chaque cellule épidermique [3] et à la désorganisation de la polarité planaire de l'aile. Ainsi, dans le mutant frizzled, il est possible de distinguer des régions au sein desquelles les poils pointent dans des directions quasi aléatoires et d'autres où les poils pointent de manière convergente dans une direction atypique. L'observation de régions au sein desquelles les poils pointent de manière conver- gente dans une direction atypique suggère qu'en l'absence d'information de polarisation planaire, les cellules restent capables de coordonner l'orientation de leur cytosquelette dans le plan de l'épithélium. Frizzled code pour un récepteur à sept domaines transmembranaires, déjà connu pour être l'un des récepteurs du ligand extracellulaire de la famille Wnt, Wingless. Cette donnée suggère donc que Frizzled participe à la réception d'un signal de polarisation planaire de nature encore inconnue [5]. L'analyse de mouches génétiquement mosaïques composées de cellules sauvages et de cellules mutantes pour frizzled indique que la mutation frizzled agit de manière non autonome au niveau cellulaire. En effet, les cellules sauvages localisées en position distale par rapport au clone de cellules homozygotes mutantes pour frizzled présentent une polarité planaire altérée (et donc un phénotype mutant). Cet effet non autonome est directionnel car les cellules sauvages localisées en position proximale par rapport au clone de cellules mutantes ont une polarité planaire intacte. Cet effet est donc qualifié de non-autonomie distale $[5,6]$. La non-autonomie distale de frizzled suggère donc que frizzled participe à la transmission d'un signal qui se propagerait des cellules proximales vers les cellules distales. Cette observation suggère en outre que les poils épidermiques sont orientés vers les cellules possédant un niveau plus faible de Frizzled. Cette hypothèse a été testée en surexprimant le gène frizzled dans la partie distale de l'aile. II en a résulté une

\begin{tabular}{|c|c|c|}
\hline Nom du gène & Structure de la protéine & Commentaires \\
\hline frizzled & $\begin{array}{c}\text { Récepteur à sept domaines } \\
\text { transmembranaires des ligands } \\
\text { de la famille Wnt }\end{array}$ & $\begin{array}{l}\text { Accumulation au pôle distal } \\
\text { des cellules de l'aile }\end{array}$ \\
\hline dishevelled & Protéine à domaines DIX, PDZ et DEP & $\begin{array}{c}\text { Accumulation au pôle distal des cellules } \\
\text { de l'aile, interagit avec Prickle et Strabismus }\end{array}$ \\
\hline prickle & Protéine à domaines PET et LIM & $\begin{array}{c}\text { Accumulation au pôle proximal des cellules } \\
\text { de l'aile, interagit avec } \\
\text { Dishevelled et Strabismus }\end{array}$ \\
\hline strabismus & Protéine à quatre domaines transmembranaires & $\begin{array}{l}\text { Accumulation au pôle proximal des cellules } \\
\text { de l'aile, interagit avec Dishevelled et Prickle }\end{array}$ \\
\hline diego & Protéine à domaines ankyrine & Accumulation aux pôles distal et proximal \\
\hline flamingo & $\begin{array}{l}\text { Protéine à sept domaines transmembranaires } \\
\text { de la superfamille des cadhérines }\end{array}$ & Accumulation aux pôles distal et proximal \\
\hline four-jointed & Protéine sécrétée & $\begin{array}{l}\text { Distribution en gradient, } \\
\text { de l'équateur au pôle, dans l'œil }\end{array}$ \\
\hline dachsous & Protéine de la superfamille des cadhérines & $\begin{array}{l}\text { Distribution en gradient, } \\
\text { du pôle à l'équateur, dans l'œil }\end{array}$ \\
\hline fat & Protéine de la superfamille des cadhérines & Distribution uniforme dans l'œil \\
\hline
\end{tabular}

Tableau I. Gènes de polarité planaire chez la drosophile. 
inversion de la polarité des poils qui, au lieu de pointer vers la partie distale de l'aile, ont poussé vers la partie proximale de l'aile qui, dans cette situation expérimentale, exprimait un niveau plus faible de frizzled [7].

La formation au pôle distal de la structure apicale qui donnera naissance au poil épidermique indique que cette région du cortex apical a des propriétés particulières. En particulier, les protéines Frizzled et Dishevelled sont localisées de manière prédominante au pôle distal du cortex apical (Figure 1, Tableau I) [8-10]. Inversement, les protéines Prickle et Strabismus sont exclues de ce domaine cortical distal et sont préférentiellement accumulées au pôle proximal du cortex apical $[11,12]$. Enfin, les protéines Flamingo et Diego présentent un enrichissement aux deux pôles [13, 14]. La localisation polarisée de ces protéines est observée aux stades durant lesquels les poils épidermiques sont formés et l'activité des gènes de polarité planaire est requise. Par ailleurs, la localisation asymétrique de chacune de ces protéines dépend de l'activité des autres gènes de polarité planaire. Ces données indiquent que la formation de domaines opposés au niveau du cortex apical des cellules épithéliales sous-tend la mise en place d'une polarité planaire.

Comment ces gènes contrôlent-ils la formation des poils au pôle distal des cellules épidermiques? Le site cortical à partir duquel le poil est formé présente une forte accumulation de microfilaments d'actine. II est donc probable que Frizzled et Dishevelled règlent l'activité et/ou la localisation de régulateurs positifs du cytosquelette d'actine. Dans de très nombreuses situations expérimentales, les petites GTPases de la famille Rho/Rac/Cdc42 jouent un rôle essentiel dans la régulation du cytosquelette d'actine. Trois études récentes indiquent que Dishevelled interagit avec une GTPase de la famille Rho via la protéine Daaml [15], et que RhoA et un effecteur de RhoA, la Rho-associated kinase de drosophile, sont requis en aval de Frizzled et Dishevelled pour restreindre la formation du poil à un seul site apical $[16,17]$. La principale cible de cette kinase lors de la polarisation planaire de l'aile semble être la chaîne régulatrice légère de la myosine (MRLC) [17]. Ces données suggèrent un modèle moléculaire simple de régulation spatiale du cytosquelette d'actine qui découle de l'activation localisée de Frizzled.

\section{Coordination de la polarisation planaire à l'échelle d'un tissu}

La localisation asymétrique des produits des gènes de polarité planaire soulève deux questions importantes.

1. Quel mécanisme permet de définir deux domaines corticaux opposés l'un à l'autre au niveau du cortex apical de chaque cellule épithéliale? Cette question importante n'est pas encore résolue. Des modèles simples basés sur les propriétés d'auto-assemblage et/ou d'exclusion réciproque de ces molécules permettent de rendre compte de la distribution unipolaire de complexes protéiques au niveau du cortex des cellules [18].

2. Quelle est l'information de position qui détermine les positions proximale et distale de ces deux domaines corticaux dans les cellules épithéliales de l'aile? Deux hypothèses générales ont été formulées pour rendre compte de l'établissement de la polarité planaire à l'échelle d'un tissu. La première hypothèse suggère qu'il existe un gradient de concentration d'une molécule $X$ (de nature inconnue) et que les cellules interprètent la différence de concentration de $« X \gg$ aux deux pôles de la cellule. L'interprétation de cette différence est supposée être indépendante de la valeur, faible ou élevée, de la concentration de «X $X$. Cette propriété est importante, car elle conditionne l'interprétation correcte de l'information de polarité planaire par les cellules proches de la source de «X» (pour lesquelles la concentration en « $X$ » est élevée) aussi bien que par les cellules qui en sont éloignées (pour lesquelles la concentration en « $X$ » est très faible). Une seconde hypothèse suggère que la polarité planaire se propage de cellules en cellules à partir d'un centre organisateur de la polarité, l'organisation du cortex d'une cellule donnée induisant ou contraignant l'organisation des cellules voisines. Ces deux modèles ne sont pas exclusifs. Aucune molécule « $X \gg$ ni aucun centre organisateur de la polarité n'ont à ce jour été clairement identifiés. Toutefois, des travaux récents ont permis d'identifier un groupe de molécules importantes pour l'organisation de la polarité planaire à l'échelle d'un tissu entier, l'œil de drosophile. Ces travaux apportent donc un éclairage moléculaire sur ces deux modèles.

\section{Polarité planaire de l'œil de drosophile}

L'œil à facettes (ou ommatidies) de la drosophile présente une polarité planaire qui est reflétée par l'arrangement en miroir, de part et autre de l'équateur qui sépare les parties ventrales et dorsales de l'œil, de deux

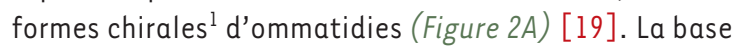
cellulaire de cette disposition en miroir des facettes est bien comprise. Le développement de l'œil la drosophile se fait par passage (du postérieur vers l'antérieur) d'un sillon morphogénétique qui déclenche la différenciation des ommatidies par recrutement successif. Au cours du développement larvaire, de petits groupes de cellules

\footnotetext{
${ }^{1}$ Formes existant sous deux configurations, images l'une de l'autre dans un miroir, et donc non superposables.
} 
différenciées photoréceptrices, d'abord symétriques et orientés de manière identique, apparaissent au sein du disque imaginal d'œil qui, à ce stade, forme un épithélium monocouche. Chacun de ces groupes de cellules subit un mouvement coordonné de rotation de $90^{\circ}$, dans le sens des aiguilles d'une montre si la future facette est localisée dans la partie dorsale du disque, ou dans le sens inverse des aiguilles d'une montre si elle se trouve en position ventrale (Figure 2A). La polarité planaire de l'œil est donc le résultat d'un mouvement de rotation des facettes au cours du développement larvaire. Le sens de rotation des facettes dépend de la position, dorsale ou ventrale, de la cellule appelée R3. Après rotation, cette cellule occupe la pointe du trapèze formé par les photorécepteurs. La cellule R3 est choisie parmi les deux cellules antérieures de l'ommatidie, avant la rotation. Ces deux cellules antérieures sont équipotentes et peuvent adopter l'identité R3 ou R4. La décision cellulaire R3/R4 dépend de l'activation différentielle du récepteur Notch. En situation sauvage, un niveau élevé d'activation de Notch est observé dans la cellule polaire (la plus éloignée de l'équateur). Cette cellule polaire adopte en conséquence l'identité R4. Inversement, un niveau plus faible d'activation de Notch est observée dans la cellule équatoriale (la plus proche de l'équateur), qui exprime un niveau élevé de Delta, le ligand de Notch. Cette cellule équatoriale adopte en conséquence l'identité R3.

Les gènes de polarité planaire décrits précédemment contrôlent la détermination des cellules R3 et R4, la rotation des facettes et la polarité planaire de l'œil (le gène strabismus tire ainsi son nom de ce défaut de polarité planaire). En contexte mutant pour frizzled, par exemple, la décision
R3/R4 n'est plus orientée dans l'espace par rapport à la ligne médiane de l'œil. II en résulte que chaque ommatidie adopte de manière aléatoire l'une ou l'autre des deux formes chirales sans tenir compte de sa position dorsale ou ventrale dans l'œil. L'analyse de mouches génétiquement mosaïques composées de cellules sauvages et de cellules mutantes pour frizzled indique qu'un niveau élevé d'activité frizzled est requis dans la cellule équatoriale. Dans cette cellule, Frizzled inhiberait Notch. Cette inhibition pourrait être relayée par Dishevelled car Dishevelled se lie à la partie intracellulaire de Notch et interfère avec l'activité de signalisation du récepteur Notch. Ainsi, une différence faible et relative d'activité Frizzled introduirait un biais spatial dans la décision R3/R4 [20-22]. Inversement, Strabismus et Prickle agiraient dans la cellule polaire pour stimuler l'activité de signalisation de Notch [21]. Les données de localisation subcellulaire des différentes protéines de polarité planaire sont en accord avec ces données génétiques. Les protéines Frizzled et Dishevelled sont accumulées dans la cellule R3 à l'interface avec la cellule R4, tandis que Prickle et Strabismus sont préférentiellement accumulées dans la cellule R4 [12, 21]. La répartition asymétrique de ces protéines à I'interface des cellules R3/R4 est formellement similaire à

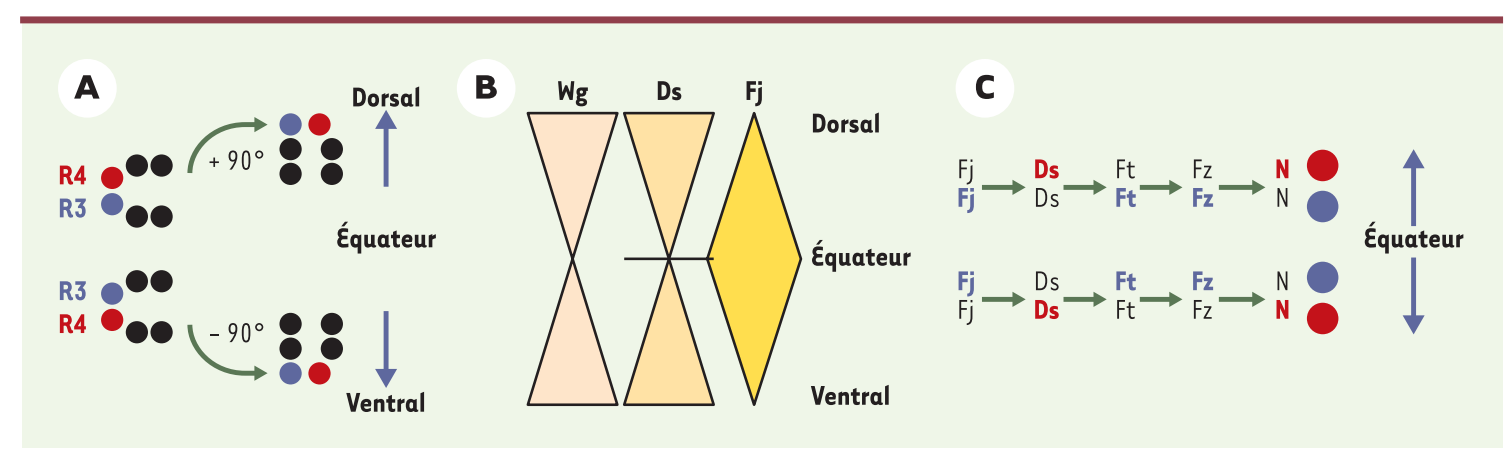

Figure 2. Polarité planaire de l'œil de drosophile. A. L'œil de la drosophile présente une polarité planaire qui résulte de la symétrie en miroir, de part et d'autre de l'équateur, de l'arrangement des deux formes chirales d'ommatidies (facettes de l'œil). Cette symétrie en miroir résulte de la rotation de $90^{\circ}$ des ommatidies. Chaque ommatidie subit un mouvement coordonné de rotation, dans le sens des aiguilles d'une montre si la future facette est localisée dans la partie dorsale du disque, ou dans le sens inverse des aiguilles d'une montre si elle se trouve en position ventrale. Le sens de rotation est imposée par la position de la cellule R3 (en bleu), qui est localisée au sommet du trapèze après rotation. Chez une mouche sauvage, avant la rotation à $90^{\circ}$, la cellule R3 (en bleu) est toujours en position équatoriale par rapport à R4 (en rouge) : elle est ventrale dans la partie dorsale de l'œil, et dorsale dans la partie ventrale de l'œil. B. Les molécules Dachsous (Ds) et Four-jointed (Fj) forment un gradient d'activité selon l'axe pôle-équateur. L'expression en gradient de Fj serait sous le contrôle du gradient de concentration de Wingless (Wg) qui agirait avec un pic de concentration décroissant des pôles vers l'équateur. C. Représentation schématique des niveaux d'activité relatifs de Fj, Ds, Fat (Ft), Frizzled ( $\mathrm{Fz}$ ) et Notch ( $\mathrm{N}$ ) dans les cellules équatoriales (bleu) et polaires (rouge). Un niveau élevé d'activité est figuré en gras et en couleur (rouge ou bleu). Les flèches indiquent le sens de la régulation telle qu'il a pu être établi génétiquement. Par exemple, un niveau élevé de Fj dans la cellule équatoriale (Fj en bleu) conduit à un niveau faible d’activité Ds dans cette même cellule (Ds en noir). Inversement, un niveau faible de Fj dans la cellule équatoriale (Fj en noir) conduit à un niveau élevé d’activité Ds dans cette même cellule (Ds en rouge). Fj pourrait soit inhiber « en cis » Ds dans la cellule équatoriale, soit activer « en trans » Ds dans la cellule polaire. Ainsi, un niveau élevé d'activité Fj au pôle conduit à une inactivation de N dans la cellule polaire, qui adopte donc l'identité R3 (en bleu). 
l'asymétrie observée dans les cellules épidermiques de l'aile (Figure 1). Ces données suggèrent fortement que la formation de domaines corticaux distincts dans le plan de la cellule sous-tend l'établissement d'une polarité planaire de l'œil.

Quelle est la nature de l'information de position qui contrôle la polarité planaire à

l'échelle du tissu ? Récemment, l'analyse de la fonction des gènes fat, dachsous et four-jointed (Tableau I) indique que ces gènes donnent une information de polarité planaire globale dans le tissu le long de l'axe équateur-pôle [23]. Le gène four-jointed code pour une protéine sécrétée exprimée en gradient de l'équateur vers le pôle [24] (Figure 2B). Les gènes fat et dachsous codent pour des protéines de la superfamille des cadhérines. Alors que Fat est distribuée de manière uniforme, la protéine Dachsous présente une distribution en gradient complémentaire du profil d'expression du gène four-jointed. L'expression en gradient de dachsous et four-jointed serait sous le contrôle du gradient de concentration de Wingless qui agirait ici comme un morphogène avec un pic de concentration allant décroissant des pôles vers l'équateur. Le gradient d'expression de dachsous résulterait d'une régulation positive par Wingless tandis que le gradient de fourjointed résulterait de l'inhibition de l'autorégulation du gène four-jointed par Dachsous [23, 24]. L'analyse de mouches génétiquement mosaïques composées de cellules sauvages et de cellules mutantes indique que ces trois gènes contrôlent la polarité planaire de l'œil [23-25] et suggère un modèle dans lequel Four-jointed inhibe Dachsous, Dachsous inhibe Fat, et Fat active Frizzled (Figure 2C) [23]. Ainsi, un gradient d'activité Four-jointed/Dachsous/Fat à l'échelle du tissu serait interprété localement au niveau de chaque cellule par Frizzled. Enfin, ces travaux suggèrent que les produits des gènes fourjointed et dachsous possèdent certaines des propriétés de la molécule « $X$ » discutée ci-dessus.

\section{Polarité planaire dans l'aile et l'abdomen chez la drosophile}

L'étude récente de la fonction des gènes fat, dachsous et four-jointed dans l'aile et dans l'abdomen montre que l'orientation globale de la polarité planaire des cellules épidermiques est déterminée par la pente des gradients d'expression des gènes dachsous et four-jointed [26, 27] (Figure 3). Les gènes fat, dachsous et four-jointed constituent ainsi une cassette fonctionnelle susceptible d'agir en aval de différents morphogènes pour établir et orienter une polarité planaire au niveau de tout un tissu en réglant l'activité d'une seconde cassette de gènes, formée des gènes frizzled, dishevelled, prickle, strabismus, flamingo ou diego, qui sont eux responsables de la mise en place d'une asymétrie planaire au sein de chaque cellule épithéliale.

\section{Polarité planaire chez les vertébrés}

Un enjeu important est de déterminer dans quelle mesure le modèle de polarisation planaire proposé chez la drosophile est transposable aux ver-

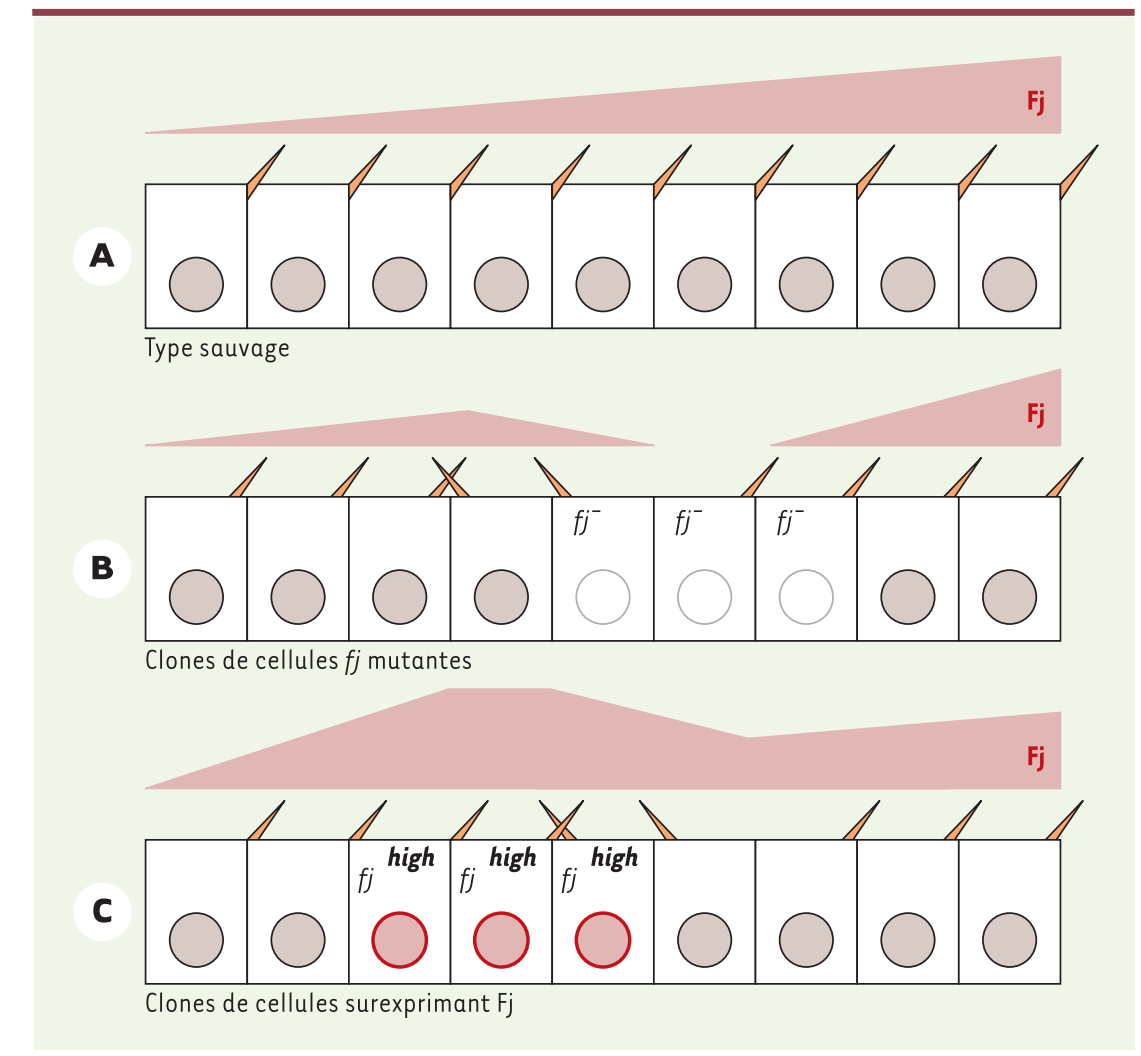

Figure 3. La pente du gradient de Four-jointed (Fj) détermine l'orientation des poils épidermiques. A. Fj est exprimé en gradient selon l'axe antéro-postérieur au sein de chaque segment abdominal. Les cellules épidermales de l'abdomen sécrètent plusieurs poils orientés vers le pôle postérieur (un seul est représenté ici par souci de simplicité). Dans le cas représenté ici, ce poil pointe vers un niveau élevé de $\mathrm{Fj}$ (en rose) au pôle postérieur. B. Les cellules mutantes et sauvages localisées à l'extrémité antérieure des clones de cellules mutantes pour fj présentent une inversion de polarité. La réorientation de ces cellules est indépendante de leur génotype. En revanche, cette réorientation reflète la pente prédite du gradient résultant de la présence du clone (en rose). En effet, la présence de cellules mutantes pour $f j$ doit modifier le gradient de $\mathrm{Fj}$, provoquant ainsi une inversion locale de la pente du gradient. C. Les cellules surexprimant $\mathrm{Fj}$ (cerclées de rouge; notées $\mathrm{Fj}$ high) ainsi que les cellules sauvages localisées à l'extrémité postérieure des clones de cellules exprimant fj présentent une inversion de polarité. Cette réorientation peut être corrélée avec la pente prédite du gradient résultant de la surexpression de $\mathrm{Fj}$ (en rose). 
tébrés et à l'homme. Des homologues des gènes frizzled, dishevelled, prickle, strabismus, flamingo ou diego ont été récemment identifiés chez les vertébrés, et des travaux récents indiquent que ces gènes pourraient agir de manière concertée pour contrôler différents mouvements cellulaires au cours de la gastrulation et de la fermeture du tube neural [15, 28-35]. Toutefois, les similitudes pouvant exister entre la polarité planaire des cellules épithéliales et la régulation de ces mouvements cellulaires ne sont pas claires. En revanche, deux études récentes montrent que des homologues de strabismus et flamingo règlent la polarité planaire dans l'oreille interne chez la souris. Chez une souris sauvage, chaque cellule auditive de l'oreille interne présente à sa surface apicale un faisceau de villosités orienté de manière stéréotypée dans le plan du tissu. Dans les souris Loop-tail et crash, mutantes respectivement pour un homologue de strabismus et de flamingo, ces faisceaux se forment mais leur orientation relative est perturbée (Figure 4) [36, 37]. Ces défauts sont formellement identiques à ceux décrits pour les mutants de polarité planaire chez la drosophile. Les gènes qui contrôlent la polarité planaire chez la drosophile pourraient donc avoir la même fonction chez les mammifères. II sera intéressant de déterminer si les produits de ces gènes sont également localisés de manière polarisée dans le plan du tissu dans ces cellules épithéliales.

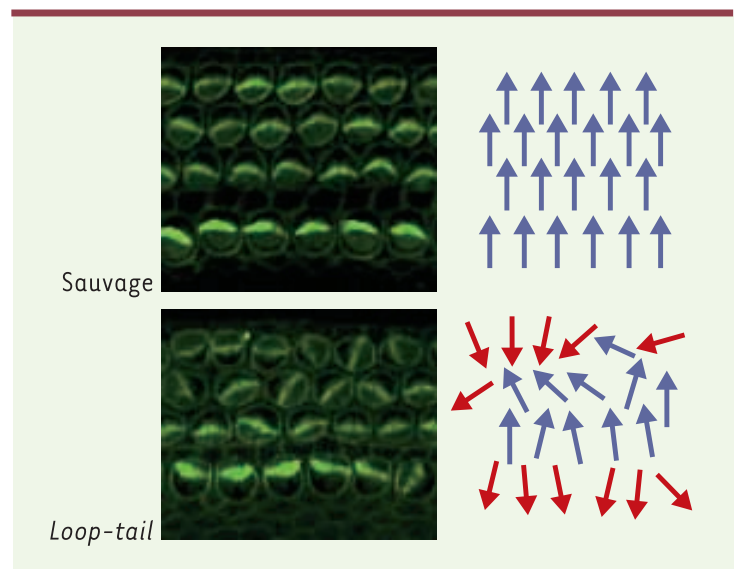

Figure 4. La mutation Loop-tail affecte la polarité planaire des cellules de l'oreille interne chez la souris. Images de surface des cellules de l'oreille interne chez une souris sauvage (en haut) ou homozygote pour la mutation Loop-tail (en bas) montrant les défauts d'orientation planaire des faisceaux de villosités présents à la surface apicale des cellules de l'oreille interne. L'orientation de chacun de ces faisceaux est figurée schématiquement par une flèche de couleur bleue (orientation correcte) ou rouge (orientation inversée) à droite des photographies. La mutation Loop-tail touche un homologue du gène strabismus de drosophile. Chez ces souris mutantes, les faisceaux se forment, mais leur orientation planaire est perturbée.

\section{Conclusions et perspectives}

L'analyse génétique de la polarité planaire chez la drosophile a permis d'identifier et de disséquer les mécanismes moléculaires qui sous-tendent la polarisation des cellules épithéliales dans le plan du tissu. Les données préliminaires obtenues chez les vertébrés confortent l'idée que ces mécanismes ont été conservés au cours de l'évolution et que le mécanisme de polarisation planaire caractérisé chez la drosophile est impliqué dans la polarisation planaire de certains épithéliums chez les mammifères. Plusieurs questions importantes restent à résoudre. II serait par exemple utile de comprendre comment Four-jointed inhibe Dachsous, comment Dachsous inhibe Fat, et comment Fat active Frizzled. De même, il semble important de comprendre comment est contrôlée la localisation asymétrique des protéines Frizzled, Dishevelled, Prickle et Strabismus, et de déterminer les cibles moléculaires de Frizzled, Dishevelled, Prickle et Strabismus qui sont impliquées dans la réalisation de la polarité planaire. Enfin, il reste à déterminer le degré de polarisation planaire des différents épithéliums chez l'homme et à évaluer le rôle physiologique de cette polarisation pour la biologie de ces épithéliums. $\diamond$

\section{REMERCIEMENTS}

Je remercie M.E. Montcouquiol pour l'image de la Figure 4 et pour la photo de couverture. Je remercie $A$. Plessis et M.E. Montcouquiol pour la relecture de cet article.

\section{SUMMARY}

\section{Genetic basis of planar polarity}

Several epitheliums exhibit a clear polarity that lies within the plane of the epithelium. This polarity, referred to as planar polarity or tissue polarity, is oriented perpendicular to the apical-basal polarity of the epithelium. Over the last two decades, the genetic and molecular bases of planar polarity have been intensively investigated in Drosophila. Recent studies have shown that establishment of planar polarity relies on the unipolar distribution of a small number of signaling molecules localizing at the apical cortex. Unipolar localization of planar polarity proteins defines two opposite and complementary cortical domains. These domains show a stereotyped orientation at the tissue level. Positioning of these cortical domains is coordinated at the tissu level by a second class of signaling molecules that form an activity gradient across the epithelium. Together these data have led to a general model of planar polarity establishment. Considering that planar polarity genes have been conserved from flies to vertebrates, this model may be useful for our understanding of epithelium biology in mammals. $\Delta$ 


\section{RÉFÉRENCES}

1. Adler PN. Planar signaling and morphogenesis in Drosophila. Dev Cell $2002 ; 2: 525-35$

2. Strutt DI. The asymmetric subcellular localisation of components of the planar polarity pathway. Semin Cell Dev Biol 2002 ; 13 : 225-31.

3. Wong LL, Adler PN. Tissue polarity genes of Drosophila regulate the subcellular location for prehair initiation in pupal wing cells. J Cell Biol $1993 ; 123: 209-21$

4. Shulman JM, Perrimon N, Axelrod JD. Frizzled signaling and the developmental control of cell polarity. Trends Genet 1998; $14: 452-8$.

5. Jones KH, Liu J, Adler PN. Molecular analysis of EMS-induced frizzled mutations in Drosophila melanogaster. Genetics 1996 ; 142 : 205-15.

6. Adler PN, Taylor J, Charlton J. The domineering non-autonomy of frizzled and van Gogh clones in the Drosophila wing is a consequence of a disruption in local signaling. Mech Dev $2000 ; 96: 197-207$.

7. Adler PN, Krasnow RE, Liu J. Tissue polarity points from cells that have higher Frizzled levels towards cells that have lower Frizzled levels. Curr Biol $1997 ; 7$ : 940-9.

8. Axelrod JD. Unipolar membrane association of Dishevelled mediates Frizzled planar cell polarity signaling. Genes Dev 2001 ; 15 : 1182-7.

9. Shimada Y, Usui T, Yanagawa S, et al. Asymmetric colocalization of Flamingo, a seven-pass transmembrane cadherin, and Dishevelled in planar cell polarization. Curr Biol $2001 ; 11: 859-63$.

10. Strutt DI. Asymmetric localization of frizzled and the establishment of cell polarity in the Drosophila wing. Mol Cell $2001 ; 7: 367-75$.

11. Bastock R, Strutt $H$, Strutt D. Strabismus is asymmetrically localised and binds to Prickle and Dishevelled during Drosophila planar polarity patterning. Development $2003 ; 130: 3007-14$

12. Tree DR, Shulman JM, Rousset $R$, et al. Prickle mediates feedback amplification to generate asymmetric planar cell polarity signaling. Cell $2002 ; 109: 371-81$

13. Feiguin F, Hannus M, Mlodzik M, et al. The ankyrin repeat protein Diego mediates Frizzled-dependent planar polarization. Dev Cell $2001 ; 1$ : 93-101.

14. Usui T, Shima Y, Shimada Y, et al. Flamingo, a seven-pass transmembrane cadherin, regulates planar cell polarity under the control of Frizzled. Cell 1999 ; 98 : 585-95.

15. Habas R, Kato Y, He X. Wnt/Frizzled activation of Rho regulates vertebrate gastrulation and requires a novel Formin homology protein Daaml. Cell $2001 ; 107: 843-54$.

16. Strutt DI, Weber U, Mlodzik M. The role of RhoA in tissue polarity and Frizzled signalling. Nature $1997 ; 387: 292-5$

17. Winter CG, Wang B, Ballew A, et al. Drosophila Rho-associated kinase (Drok) links Frizzled-mediated planar cell polarity signaling to the actin cytoskeleton. Cell 2001; $105: 81-91$.

18. Meinhardt H. Orientation of chemotactic cells and growth cones: models and mechanisms. J Cell Sci $1999 ; 112: 2867-74$.

19. Strutt H, Strutt D. Polarity determination in the Drosophila eye. Curr Opin Genet Dev 1999 ; 9 : 442-6.

20. Fanto M, Mlodzik M. Asymmetric Notch activation specifies photoreceptors R3 and R4 and planar polarity in the Drosophila eye. Nature 1999 ; 397 : 523-6.
21. Strutt D, Johnson R, Cooper K, et al. Asymmetric localization of Frizzled and the determination of Notch-dependent cell fate in the Drosophila eye. Curr Biol 2002 ; 12 : 813-24.

22. Cooper MT, Bray SJ. Frizzled regulation of Notch signalling polarizes cell fate in the Drosophila eye. Nature 1999 ; 397 : 526-30.

23. Yang $\mathrm{CH}$, Axelrod JD, Simon MA. Regulation of Frizzled by fat-like cadherins during planar polarity signaling in the Drosophila compound eye. Cell $2002 ; 108: 675-88$

24. Zeidler MP, Perrimon N, Strutt DI. The four-jointed gene is required in the Drosophila eye for ommatidial polarity specification. Curr Biol 1999 ; 9: 1363-72.

25. Rawls AS, Guinto JB, Wolff T. The cadherins fat and dachsous regulate dorsal/ventral signaling in the Drosophila eye. Curr Biol 2002 ; 12 : 1021-6.

26. Casal J, Struhl G, Lawrence P. Developmental compartments and planar polarity in Drosophila. Curr Biol 2002; 12 : 1189

27. Ma D, Yang $\mathrm{CH}, \mathrm{McNeill} \mathrm{H}$, et al. Fidelity in planar cell polarity signalling. Nature $2003 ; 421: 543-7$.

28. Darken RS, Scola AM, Rakeman, AS, et al. The planar polarity gene strabismus regulates convergent extension movements in Xenopus. EMBO J $2002 ; 21: 976-85$

29. Goto T, Keller R. The planar cell polarity gene strabismus regulates convergence and extension and neural fold closure in Xenopus. Dev Biol $2002 ; 247: 165-81$

30. Jessen JR, Topczewski J, Bingham S, et al. Zebrafish trilobite identifies new roles for Strabismus in gastrulation and neuronal movements. Nat Cell Biol $2002 ; 4: 610-5$.

31. Kibar Z, Vogan KJ, Groulx N, et al. Ltap, a mammalian homolog of Drosophila Strabismus/Van Gogh, is altered in the mouse neural tube mutant Loop-tail. Nat Genet $2001 ; 28: 251-5$.

32. Myers D, Sepich D, Solnica-Krezel L. Convergence and extension in vertebrate gastrulae: cell movements according to or in search of identity? Trends Genet $2002 ; 18: 447$.

33. Wallingford JB, Rowning BA, Vogeli KM, et al. Dishevelled controls cell polarity during Xenopus gastrulation. Nature 2000 ; 405 : 81-5.

34. Wallingford JB, Goto T, Keller R, et al. Cloning and expression of Xenopus Prickle, an orthologue of a Drosophila planar cell polarity gene. Mech Dev 2002 ; 116 : 183-6.

35. Wallingford JB, Fraser SE, Harland RM. Convergent extension: the molecular control of polarized cell movement during embryonic development. Dev Cell $2002 ; 2: 695-706$

36. Curtin JA, Quint $\varepsilon$, Tsipouri V, et al. Mutation of Celsrl disrupts planar polarity of inner ear hair cells and causes severe neural tube defects in the mouse. Curr Biol 2003; $13: 1129-33$.

37. Montcouquiol M, Rachel RA, Lanford PJ, et al. Identification of Vangl2 and Scrbl as planar polarity genes in mammals. Nature $2003 ; 423: 173-7$.

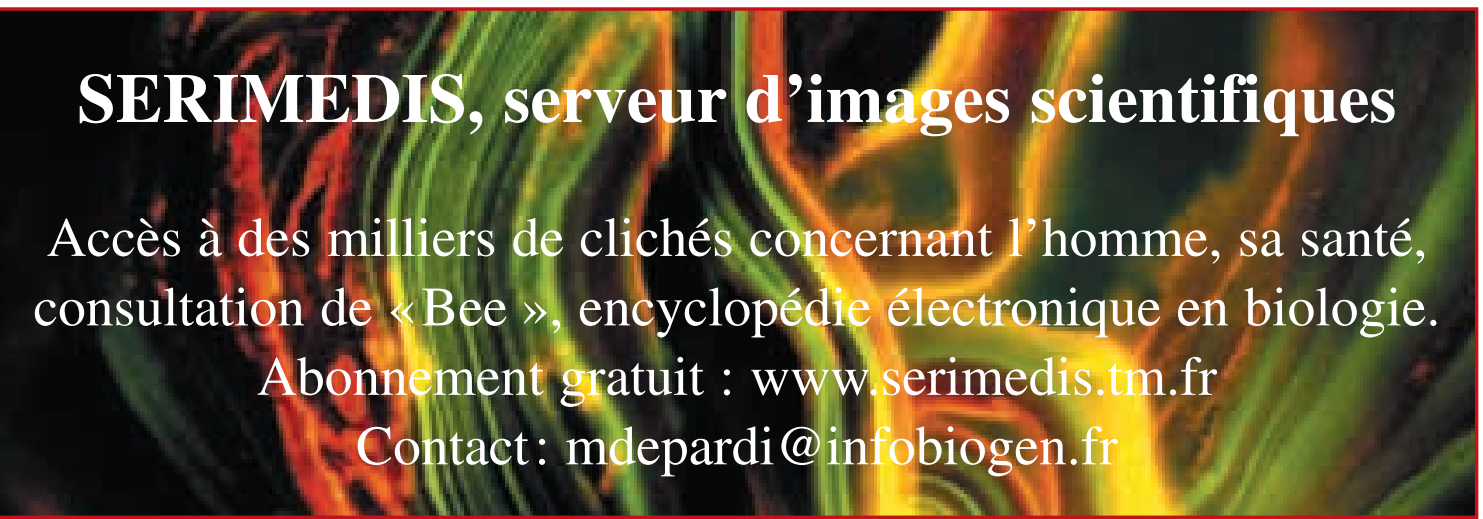

TIRÉS À PART

F. Schweisguth 